their ionisation states and to deduce the mobilities of the side chains of certain residues.

T. G. Spiro (Princeton University) showed how resonance Raman spectroscopy of haem proteins can be used to detect deviations from planarity of the porphoryin ring and other structural consequences of changing the spin and oxidation states in the haem groups. The vibrational modes containing this information exhibit greatly enhanced Raman scattering when an electronic transition to which they are coupled is irradiated with laser excitation.

X-ray and neutron scattering techniques were considered by J. E. Enderby (University of Leicester) and J. W. White (University of Oxford). Because the neutron diffraction scattering length for the proton is opposite in sign to that for deuterium, by using mixtures of $\mathrm{H}_{2} \mathrm{O} / \mathrm{D}_{2} \mathrm{O}$ it is possible to achieve high contrast between the scattering from the solvent and from the solute molecules. For inelastic scatter- ing there is a large difference in cross section between protons and deuterons which allows observation of the dynamic behaviour of individual components in the various phases of amphiphile/water systems. Low angle neutron diffraction studies on hydrated collagen and tobacco mosaic virus solutions lead to information about the radii of gyration of the molecules in solution.

From measurements of the quasielastic spectrum of laser light scattered from macromolecules in solution, G. B. Benedek (Massachusetts Institute of Technology) showed how accurate diffusion coefficients can be obtained. When considered together with sedimentation data these values provide molecular weights. The size and shape of the macromolecules can also be deduced. Interesting differences in the apparent volumes of lysozyme when denatured by different methods (guanidine and heat) were observed but no evidence for intermediate states in the unfolding process could be detected.

\section{Optical systems for space}

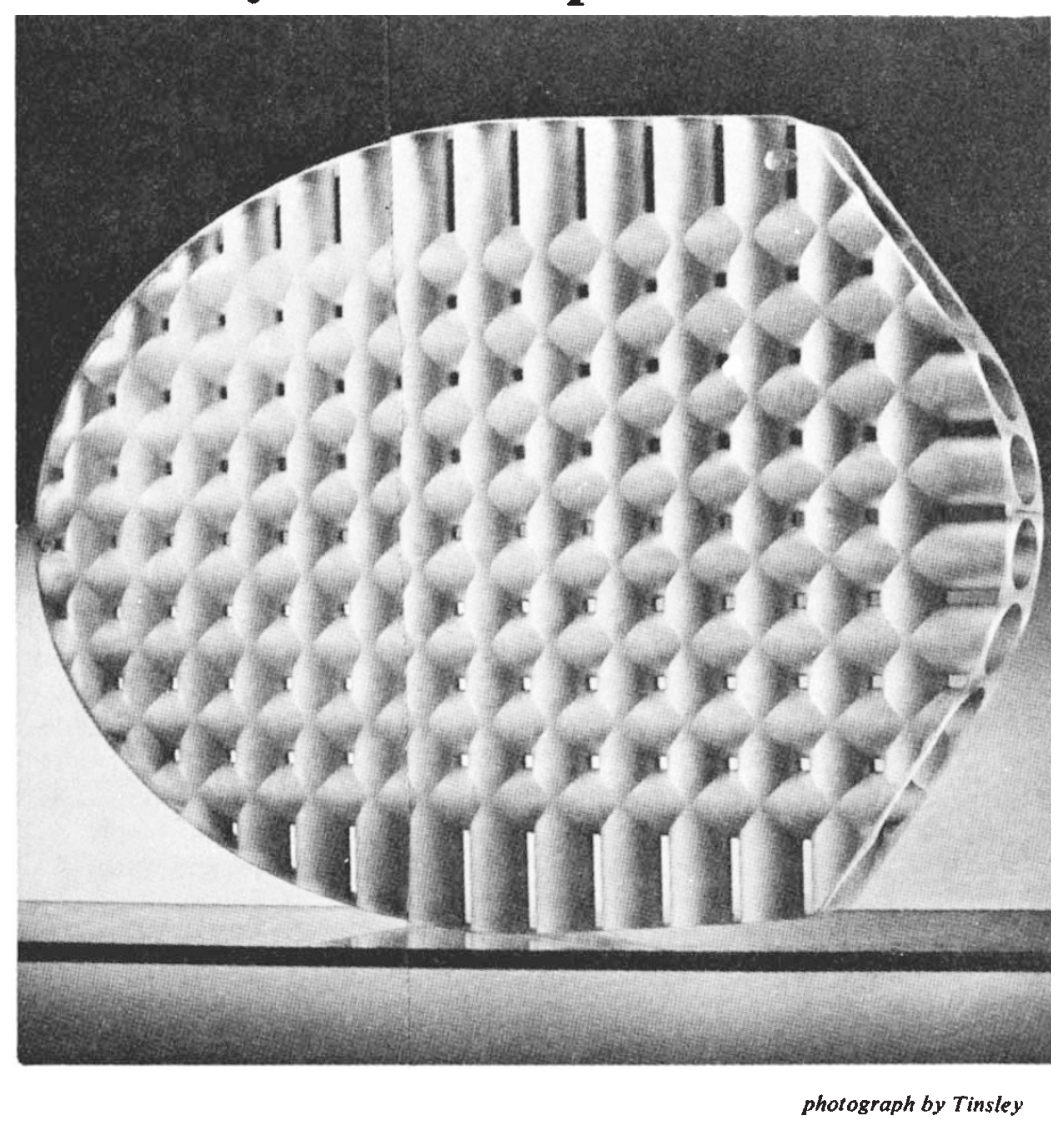

The mirror illustrated (made by Tinsley in Berkeley, California) is designed to be part of a rocket-launched optical system. The grid pattern is formed by a series of holes drilled through the core to minimise weight. Because of the high stresses experienced in a large object during a launch, the accurasy of machining is critical and is evident in the regularity of intersection of the holes.
The technique is capable of monitoring aggregation of macromolecules and this was well illustrated by a study of the manner in which the aggregation of glutamate dehydrogenase controls the amount of enzyme in its active form.

Most of the techniques considered have still to reach their full potential in their application to structure determination in solution and rapid progress can be anticipated over the next few years.

\section{Huntingtons's chorea and GABA}

from T. J. Crow

THE apparent diversity of chemical neurotransmitter substances (there are now at least seven with good credentials) in the brain has encouraged the search for specific neurohumoural disturbances underlying neurological diseases of obscure aetiology. Ehringer and Hornykiewicz' discovery (Klin. Wschr., 38, 1236-1239; 1960) of a deficiency of dopamine in the basal ganglia in Parkinson's disease led to the introduction of L-DOPA in treatment. Now it is suggested that another progressive disease of the 'extrapyramidal' motor system, Huntington's chorea, is associated with a specific deficiency of GABA ( $\gamma$-amino-butyric acid) in the same brain regions.

GABA, known for 20 years to be present in brain, has a regional distribution which follows that of its synthetic enzyme glutamine acid decarboxylase (GAD), and both are present in synaptosomal fragments. GABA is in high concentration within the corpus striatum, globus pallidus and substantia nigra, structures within the extrapyramidal motor system, and also within the cerebellar cortex and nuclei (Fahn and Coté, J. Neurochem., 15, 209-213; 1968). The presence of a high-affinity uptake system and the demonstration of release following neural stimulation provide further evidence that this substance has a neurotransmitter role, and microionophoretic data suggest that at several sites GABA is an inhibitory transmitter. The recent development of an immunohistochemical technique by Saito et al. (Proc. natn. Acad. Sci. U.S.A., 71, 269-273; 1974) for GAD may greatly facilitate the task of localising particular gabaminergic systems.

Huntington's chorea is transmitted as an autosomal dominant condition which may affect between 4 and 7 per 100,000 of the population. The disease owes its persistence, and its peculiarly sinister character, to the fact that the average age of onset (44 years, according to Wendt) is beyond the normal period of fertility. Thus by the time symptoms appear the patient may have 